\title{
Indirect Tire Pressure Monitoring Using Sensor Fusion
}

\author{
Niclas Persson, Fredrik Gustafsson, Markus Drevo \\ Division of Communication Systems \\ Department of Electrical Engineering \\ Linköpings universitet, SE-581 83 Linköping, Sweden \\ WWW: http://www. control.isy.liu.se \\ E-mail: persson@isy.liu.se, fredrik@isy.liu.se \\ markus.drevo@niradynamics.se
}

22nd October 2002

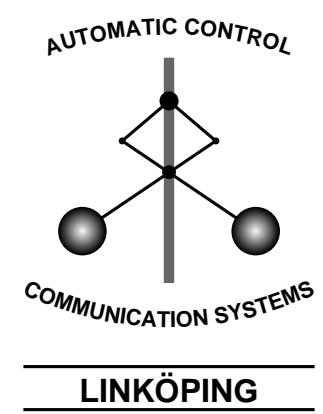

Report no.: LiTH-ISY-R-2471

Submitted to SAE'02, Detroit, MI, USA

Technical reports from the Control \& Communication group in Linköping are available at http://www. control.isy.liu.se/publications. 


\begin{abstract}
Vehicle handling depends critically on the tire-road contact patch. When the tire inflation pressure changes the contact patch is no longer optimal and the handling properties deteriorate. Furthermore fuel consumption increases and the lifetime of the tires decreases. Therefore it is very important that the tires are correctly inflated.

We here focus on an indirect tire pressure monitoring system, where no pressure sensors are needed. The system is based on vibration and wheel radius analysis. These two approaches are combined for optimal performance concerning sensitivity to detect pressure losses and robustness to different driving conditions. When these two approaches are combined, it is possible to detect pressure losses larger than $15 \%$ in one, two, three, or four (diffusion) tires within 1 minute. It is also possible to detect which of the tires that are underinflated.
\end{abstract}

Keywords: Tire, Pressure, Monitoring, Sensor, Fusion, Indirect 


\title{
Indirect Tire Pressure Monitoring Using Sensor Fusion
}

\author{
Niclas Persson, Fredrik Gustafsson \\ Department of Electrical Engineering, Linköping University \\ Markus Drevö \\ Nira Dynamics AB
}

Copyright (@ 2002 Society of Automotive Engineers, Inc.

\begin{abstract}
Vehicle handling depends critically on the tire-road contact patch. When the tire inflation pressure changes the contact patch is no longer optimal and the handling properties deteriorate. Furthermore fuel consumption increases and the lifetime of the tires decreases. Therefore it is very important that the tires are correctly inflated.

We here focus on an indirect tire pressure monitoring system, where no pressure sensors are needed. The system is based on vibration and wheel radius analysis. These two approaches are combined for optimal performance concerning sensitivity to detect pressure losses and robustness to different driving conditions. When these two approaches are combined, it is possible to detect pressure losses larger than $15 \%$ in one, two, three, or four (diffusion) tires within 1 minute. It is also possible to detect which of the tires that are underinflated.
\end{abstract}

\section{INTRODUCTION}

The importance of correct tire inflation pressure is well known. Vehicle handling is decided upon the tire-road contact patch. Furthermore fuel consumption increases and tire lifetime decreases with $20 \%$ for every 0.2 bar under-inflation. It is also known [11] that tire problems are the third most common breakdown for passenger vehicles. $75 \%$ of all tire flats are preceded by underinflation or slow leakage. With a tire pressure monitoring system severe accidents can be avoided and both economical and environmental benefits can be made.

There are two ways of monitoring the tire pressure. One way is to mount a pressure sensor at the rim on each tire and via a communication-link (usually a radio-link) transfer the sensor value to a central unit. This is called direct tire pressure monitoring. Due to the extra hardware needed this method is very expensive. The other alternative, called indirect tire pressure monitoring uses existing sensors and software algorithms. The indirect systems are very cost-effective (no extra hardware).

There are plenty of on-going R\&D projects (see e.g. [12]) on indirect Tire Pressure Monitoring Systems (TPMS), reflected in over 100 patents. There are two classes of indirect TPMS.

1. Vibration analysis using the fact that the rubber in the tire reacts like a spring when excited by road roughness. The vibration analysis can be performed by FFT-based methods or by parametric methods (using an auto-regressive model). The idea is to monitor the resonance frequency, which is correlated with the tire pressure (see e.g. [5] and [6]).

2. Wheel radius analysis using the fact that the tire pressure affects the effective rolling radius of the tire. The most common suggestion is to monitor a residual, based on a static non-linear transformation of wheel speeds, which should be close to zero when the tires are equally large, i.e. have equally tire pressure (see e.g. [7]).

Most of the on-going R\&D projects are in the latter class. These two classes have some different properties regarding robustness and sensitivity, for instance to varying velocity, and ability to detect pressure changes. Typically wheel radius analysis is very sensitive to different velocities, but on the other hand it responds very quickly to pressure changes. The vibration analysis is sensitive to different road conditions, but is insensitive to various velocities.

The idea here is to combine these two classes in a way such that both robustness and sensitivity properties are enhanced. The method proposed for the wheel radius analysis uses both longitudinal and lateral vehicle dynamic models. The longitudinal model compares the driven and non-driven wheels, using a friction model, while the lateral model compares the left and right wheels, using a yaw rate model. The analysis is based on [1], [2] and [3]. The vibration analysis includes a preprocessing unit, see [4], to improve the signal quality from the ABS-sensors, which makes the analysis 
feasible. This method uses ABS-sensor signals only to monitor the tire pressure.

\section{TIRE MODEL}

The information sources for indirect TPMS are wheel speed sensors and perhaps inertial sensors like rategyros and accelerometers. The main sources are the wheel speed sensors; in modern vehicles these sensors are integrated in the ABS-system.

The tire can be modeled as a spring-damper system both in vertical and torsional direction, see Figure 1.

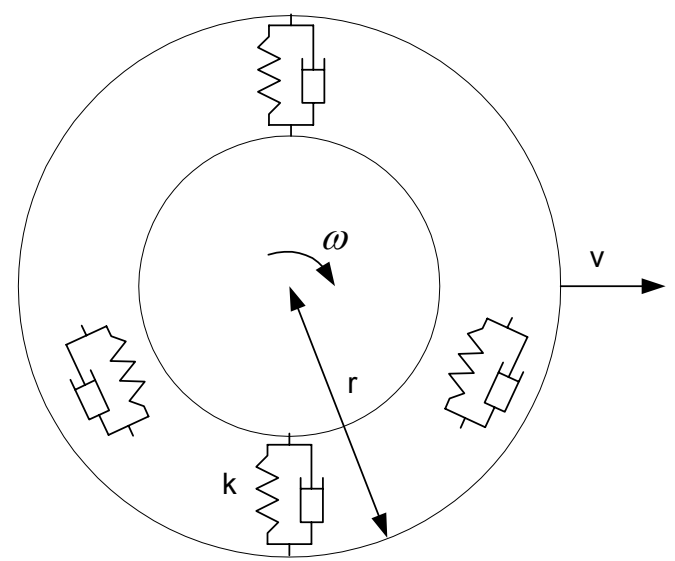

Figure 1: Tire modeled as spring-damper system in both vertical and torsional direction.

The vertical spring-damper system is the source of two phenomena. First, the effective rolling radius is dependent on the inflation pressure and if the pressure is decreased the radius is decreased. This causes the tire to rotate faster. In the equation $\delta_{r}$ denotes the change of radius affected by deflation.

$$
\omega=\frac{v}{r-\delta_{r}}
$$

Secondly, the tire vibrates in the vertical direction excited by road roughness and causes the effective rolling radius to fluctuate with a specific resonance frequency. Indirectly this results in a fluctuation of the wheel speed at the same resonance frequency. When the tire inflation pressure decreases the spring-constant is decreased, $\Delta_{k}$, and this yields a lower resonance frequency. The most significant mode for this vibration is about $10-20 \mathrm{~Hz}$.

$$
\omega_{\text {resonance }}=\sqrt{\frac{k-\Delta_{k}}{N}}
$$

In the equation $N$ denotes the normal force applied to the wheel. The torsional spring-damper system is also excited by road roughness. The tire vibrates in the torsional direction and the vibration directly affects the wheel speeds. Again, the vibration frequency is decreased when the tire inflation pressure is decreased. For this vibration the most significant vibration mode is about $40-50 \mathrm{~Hz}$.

\section{TPMS USING VIBRATION ANALYSIS}

The idea here is to monitor the vibration frequency from the vertical and torsional spring-damper systems and to detect abnormal values of the frequency. Here we have only used the torsional vibration. The frequency content (FFT) of the wheel speed signal for three different test runs (with different pressure) is shown in Figure 2. As is evident from the figure the correlation between resonance peak frequency and inflation pressure can be used for tire pressure monitoring. In the figure, batches of 30 seconds test runs are used.

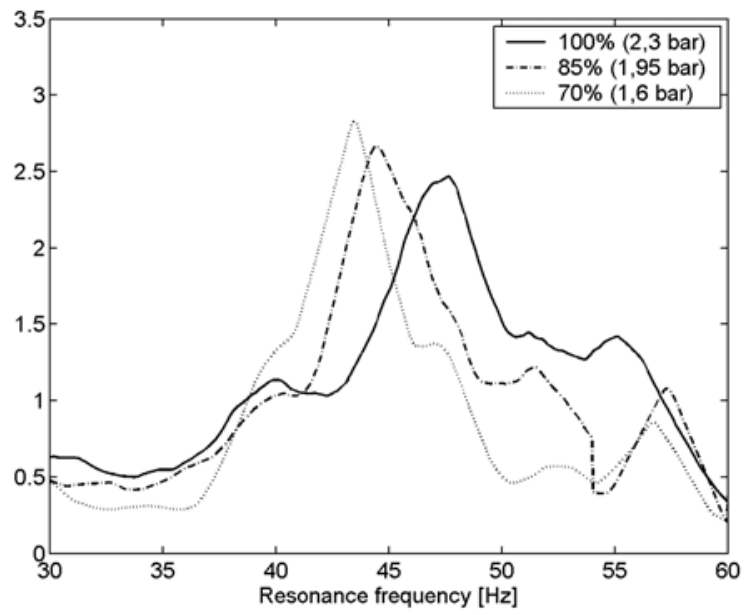

Figure 2: Smoothed FFT of wheel speed signal for three different test runs with $100 \%, 85 \%$ and $70 \%$ of the nominal inflation pressure, respectively.

The FFT is a batch-wise data processing and consumes a large amount of memory and causes a time delay. An alternative is to estimate the resonance frequency recursively using a second order damper-spring model of the form

$$
y_{t}=\frac{1}{1+a_{1} q^{-1}+a_{2} q^{-2}}
$$

where $y_{t}$ is the wheel speed and $q$ is the shift operator $q^{-1} y_{t}=y_{t-1}$. Model-based parameter estimation can easily be implemented with standard recursive methods, such as recursive least squares (RLS) or Kalman filter. The parameters $a_{1}$ and $a_{2}$ are transformed by means of a non-linear function to the frequency value of the resonance peak. Using a recursive filter, the resonance peak frequency becomes a function of time and is shown in Figure 3. 


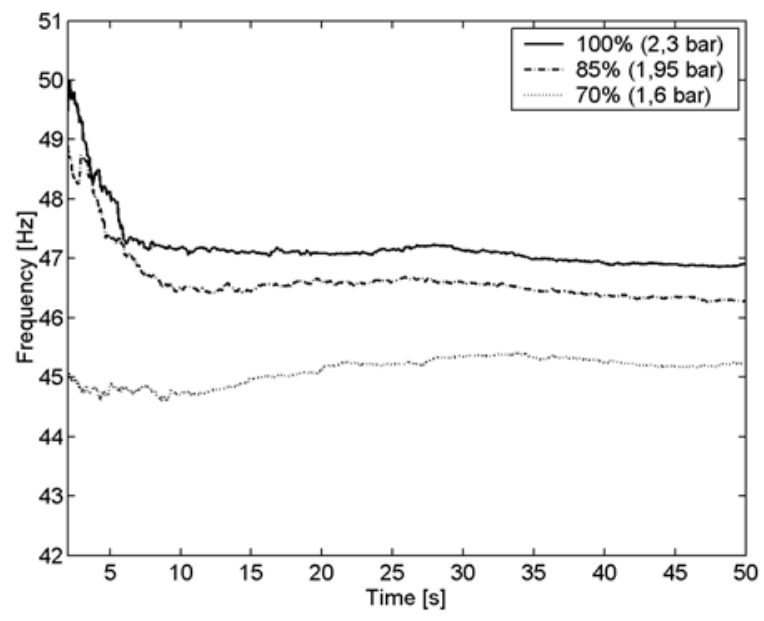

Figure 3: Recursive estimates of torsional vibration frequency for three different test runs with $100 \%, 85 \%$ and $70 \%$ of the nominal inflation pressure, respectively.

To evaluate the performance of the filter, 30 test runs were performed. The first 10 have nominal pressure, the ten in the middle are $15 \%$ under-inflated and the last 10 are $30 \%$ under-inflated. The test runs include different driving styles, surfaces and velocities. An average of the recursive estimate for each test run is shown in Figure 4. A pressure decrease of $15 \%$ is detectable within 30 seconds, but a more realistic threshold is $25 \%$ within one minute.

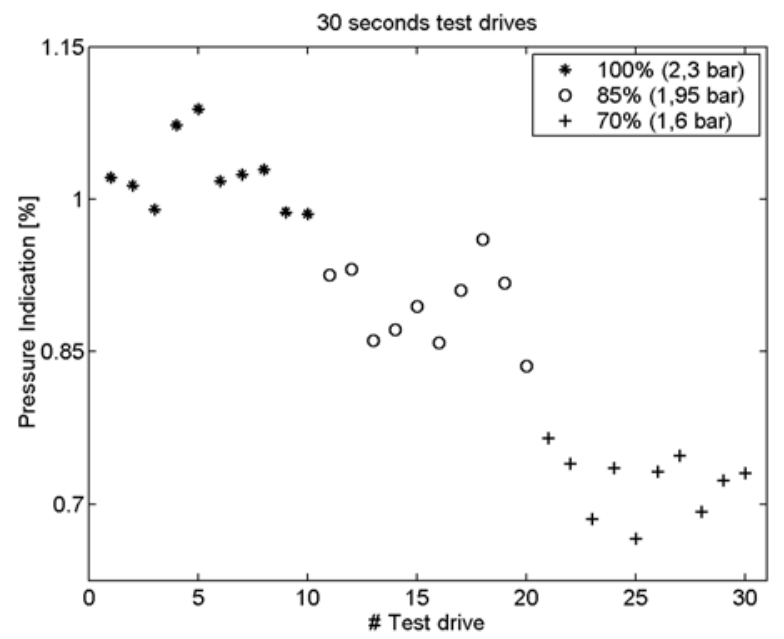

Figure 4: Average of pressure indication for 30 test runs with $100 \%, 85 \%$ and $70 \%$ of the nominal inflation pressure, respectively. The pressure indication is the normalized vibration frequency.

Vibration analysis has the advantage to independently evaluate every tire and therefore it is also possible to detect pressure losses in all four tires (diffusion).

\section{TPMS USING WHEEL RADIUS ANALYSIS}

TPMS based on wheel radius analysis uses the fact that the effective rolling radius of the wheel decreases if the tire loses pressure. This is perhaps the most intuitive way of detecting under-inflation by monitoring wheel speeds, but it has both advantages and disadvantages.

Most of the existing approaches to TPMS use static nonlinear relations to detect under-inflation. One example is

$$
r=\frac{\omega_{1}}{\omega_{2}}-\frac{\omega_{3}}{\omega_{4}}
$$

The wheel speeds $\omega_{i}$ are enumerated as front left (1), front right (2), rear left (3), and rear right (4). This enumeration is used in the rest of the paper. A pressure loss is detected when the test statistic, $r$, is non-zero. As an inherit disadvantage, this static consistency test cannot detect equal pressure losses on the same axle or side. Assuming that all tires are traveling with the same velocity this is seen from

$$
r=\frac{\omega_{1}}{\omega_{2}}-\frac{\omega_{3}}{\omega_{4}}=\frac{r_{2}}{r_{1}}-\frac{r_{4}}{r_{3}}=\frac{r_{2} r_{3}-r_{1} r_{4}}{r_{1} r_{3}} .
$$

An equal pressure loss in for example both rear tires does not affect the test statistic, $r$. Instead of using a static non-linearity, we use both longitudinal and lateral vehicle dynamics. The longitudinal dynamics compares the driven and non-driven wheel speeds, using a friction model, while the lateral dynamics compares left and right wheel speeds, using a yaw rate model.

\section{LONGITUDINAL DYNAMICS}

As suggested in [8] a friction model can be used to detect under-inflation. The friction model is based on the linear part of the classic longitudinal slip model, Figure 5, described in for instance [9] and [10]. The wheel speeds of front (driven) and rear (non-driven) wheels are compared to compute the slip.

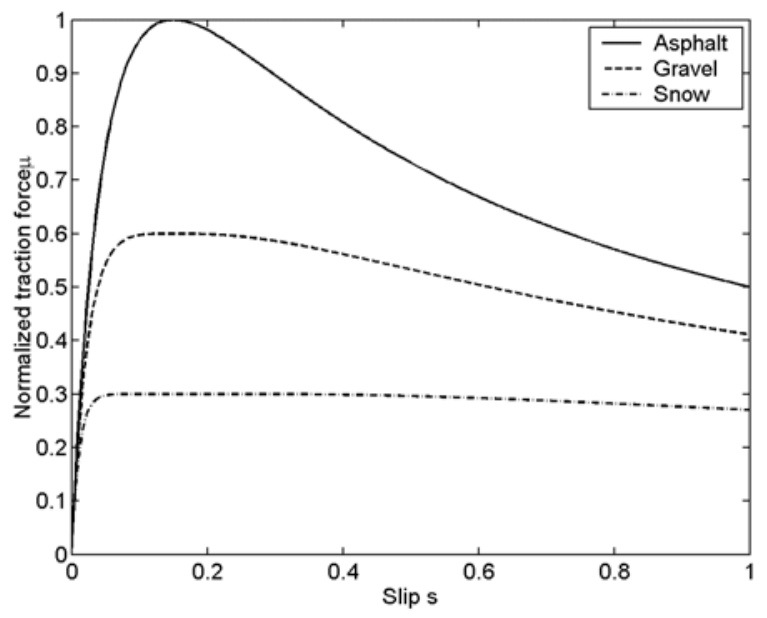

Figure 5: Slip curves for asphalt, gravel and snow. The slope of the linear part ( $s$ small) is used in the TPMS. 
The wheel speed and engine torque are computed from standard sensors. The wheel slip is defined as the difference between the circumferential and longitudinal velocity.

$$
s=\frac{\omega r-v_{x}}{v_{x}}
$$

For small values of the normalized traction force, $\mu=F_{x} / F_{z}$, the wheel slip is a linear function of the normalized traction force and the relative difference in tire radius between the front and rear wheel

$$
s=\mu / k+\delta
$$

where $k$ is the inverse of the longitudinal stiffness and $\delta$, for the left side, is defined as

$$
\delta_{13}=1-\frac{r_{1}}{r_{3}}
$$

Using the state vector $x_{t}=\left(\begin{array}{ll}1 / k_{l} & \delta_{13}\end{array}\right)^{T}$ the discrete time state space model for the left side of the vehicle is

$$
\begin{aligned}
& x_{t+1}=x_{t}+v_{t} \\
& s_{t}=\left(\begin{array}{ll}
\mu_{l, t} & 1
\end{array}\right) x_{t}+e_{t}
\end{aligned}
$$

An analogous and independent model is used for the right side of the vehicle. The offset, $\delta$, is caused by unequal tire radius and can be used in the TPMS as an indicator of the tire inflation pressure. The state space model can be directly applied to a Kalman filter and with proper tuning a decent trade-off between noise attenuation and tracking speed can be achieved.

\section{Experimental results}

In order to evaluate the performance of estimating $\delta_{13}$ (left side) and $\delta_{24}$ (right side), a series of test runs were conducted. The test runs included 5 different scenarios.

1. Nominal pressure in all tires.

2. Low pressure in front left from the beginning.

3. Low pressure in front left after 220 seconds.

4. Low pressure in rear left from the beginning.

5. Low pressure in rear left after 220 seconds.

The result is shown in Figure 6 . The response to the pressure change is fast and accurate and it takes only about 60 seconds to track the right level.
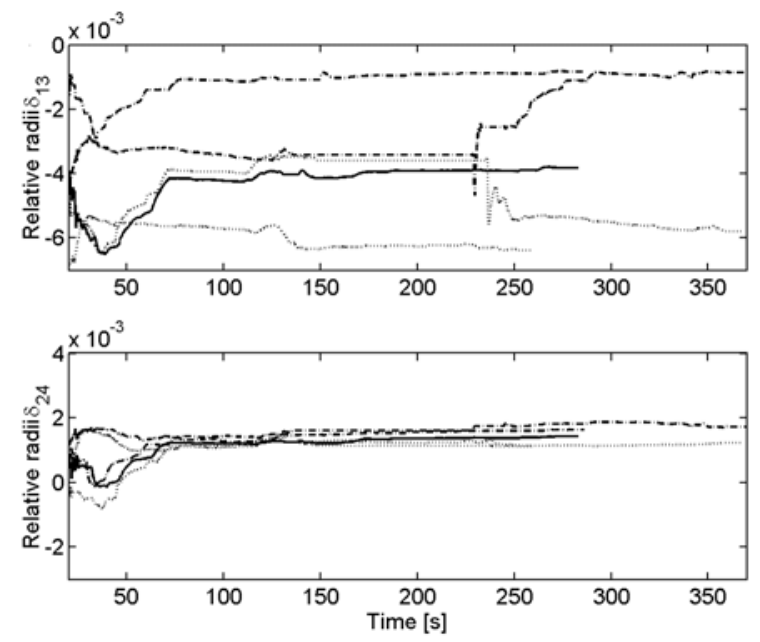

Figure 6: Recursive estimates of longitudinal dynamic relative radius parameters. The solid line represents nominal pressure; the dash dotted low pressure in front left and the dotted low pressure in rear left.

\section{LATERAL DYNAMICS}

Instead of comparing front and rear tires, left and right tires can be compared. Additional to wheel speed sensors a rate gyro is needed. Rate gyros have become more and more common in modern vehicles due to the fact that the number of stability assistant systems increases and they demand rate gyros.

Using a lateral dynamic model it is possible to compute the yaw rate from wheel angular speeds.

\section{Dynamic model}

The well-known relation between yaw rate $\dot{\psi}$, longitudinal velocity $v_{x}$ and curve radius $R$ (see e.g. [9] and [10]) is

$$
\dot{\psi}=\frac{v_{x}}{R}
$$

The velocity at the center of the rear axle is

$$
v_{x}=\frac{v_{3}+v_{4}}{2}
$$

The curve radius is defined as the distance between the center of the rear axle and the Instantaneous Center of Motion, ICM.

$$
R^{-1}=\frac{2\left(v_{3}-v_{4}\right)}{L_{\text {rear }}\left(v_{3}+v_{4}\right)}
$$


With the definition $\delta_{34}=\frac{r_{3}}{r_{4}}-1$ and with $L_{\text {rear }}$ denoting the distance between the rear wheels, yaw rate is computed as

$\dot{\psi}=\frac{\left(v_{3}-v_{4}\right)}{L_{\text {rear }}}=\frac{\left(\omega_{3} r_{3}-\omega_{4} r_{4}\right)}{L_{\text {rear }}}=\omega_{4} r_{4} \frac{\frac{\omega_{3}}{\omega_{4}} \delta_{34}+\frac{\omega_{3}}{\omega_{4}}-1}{L_{\text {rear }}}$ The tire radius $r_{4}$ is unknown and a good working approximation is to use the nominal tire radius $r_{n}$.

$\dot{\psi}=\frac{\omega_{3} r_{n}}{L_{\text {rear }}} \delta_{34}+r_{n} \frac{\omega_{3}-\omega_{4}}{L_{\text {rear }}}=\frac{\omega_{3} r_{n}}{L_{\text {rear }}} \delta_{34}+\dot{\psi}_{\text {m,rear }}$

where $m$ indicates a computable value. Again, an analogous model is used for the front axle.

The gyro signal is subject to an offset error and needs to be modeled. A good working approximation of gyro imperfections is

$$
\dot{\psi}_{\text {gyro }}=\dot{\psi}+\delta_{o} .
$$

\section{$\underline{\text { Kalman filter }}$}

A state space model for the lateral dynamic models (front and rear) and gyro model uses the state vector

$$
x=\left(\begin{array}{lllll}
\dot{\psi} & \ddot{\psi} & \delta_{o} & \delta_{34} & \delta_{12}
\end{array}\right)^{T}
$$

and the measurements are arranged as

$$
y_{t}=\left(\begin{array}{lll}
\dot{\psi}_{\text {gyro }} & \dot{\psi}_{m, \text { rear }} & \dot{\psi}_{m, \text { front }}
\end{array}\right)^{t}
$$

With these two vectors a discrete time state space model can be derived as

$$
\begin{aligned}
& x_{t+1}=\left(\begin{array}{ccccc}
1 & T s & 0 & 0 & 0 \\
0 & 1 & 0 & 0 & 0 \\
0 & 0 & 1 & 0 & 0 \\
0 & 0 & 0 & 1 & 0 \\
0 & 0 & 0 & 0 & 1
\end{array}\right) x_{t}+\left(\begin{array}{c}
T_{s}^{2} / 2 \\
T_{s} \\
0 \\
0 \\
0
\end{array}\right) v_{t} \\
& y_{t}=\left(\begin{array}{ccccc}
1 & 0 & 1 & 0 & 0 \\
1 & 0 & 0 & -r_{n} \frac{\omega_{3}}{L_{\text {rear }}} & 0 \\
1 & 0 & 0 & 0 & -r_{n} \frac{\omega_{1}}{L_{\text {front }}}
\end{array}\right) x_{t}+e_{t}
\end{aligned}
$$

It is here assumed that the unknown input $v_{t}$ only affects the yaw acceleration, which is common for motion models. The discrete time model is applied by a Kalman filter and the tire inflation pressure correlated parameters $\delta_{12}$ and $\delta_{34}$ can be estimated.

\section{Experimental results}

Figure 7 illustrates the estimated $\delta$-parameters for the lateral dynamics model. The same test runs as in the longitudinal dynamics are used. Again, the tracking is both fast and reliable. The same performance as for the longitudinal dynamics is achieved. As can be seen the estimated parameters are non-zero even with nominal pressure in all tires. The reason for this is different wear and tear for all tires. A calibration routine is needed to compute the nominal level of the estimated parameters.
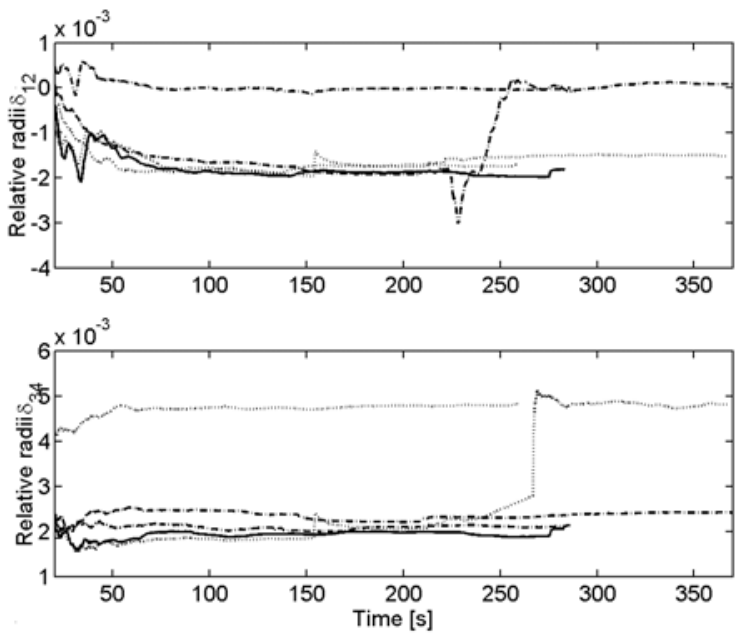

Figure 7: Recursive estimation of lateral dynamics relative radius parameters for the 5 test runs defined above. The solid line represents nominal pressure; the dash dotted low pressure in front left and the dotted low pressure in rear left.

\section{FUSION OF VIBRATION AND WHEEL RADIUS ANALYSIS}

Both vibration and wheel radius analysis carry information about the current tire inflation pressure. They can be used independently, but for optimal performance they should be combined. Wheel radius analysis estimates the relative radii between front, rear, left and right wheel pairs, respectively. Using simple logistic it is possible to detect whether one, two or three tires are under-inflated. Both vibration and wheel radius analysis need calibration after the pressure is changed in one or more tires. The calibration routine computes the nominal value of the resonance frequency and relative tire radius. Neither of the proposed methods includes a state for the absolute tire pressure and the standard fusion formula cannot be applied. Instead a voting scheme has to be applied. 


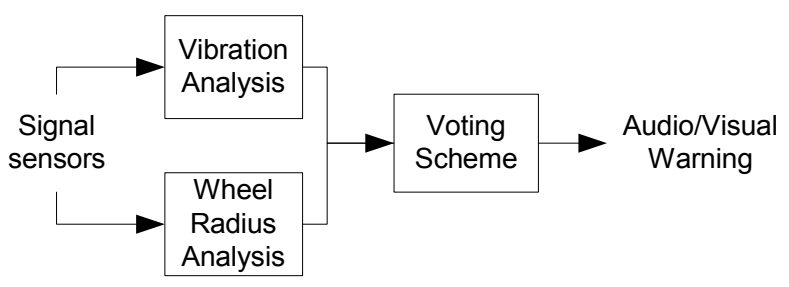

Figure 8: A voting scheme is used for the fusion of vibration and wheel radius analysis.

Both vibration and wheel radius analysis deliver two signals.

1. A flag $t_{i}(i=1,2)$ indicating which tire is/are under-inflated.

2. A confidence $c_{i}(i=1,2)$ level of the first signal.

The voting scheme works as follows:

If both flags indicate the same under-inflation, $t_{1}=t_{2}$, the mean of the confidence levels is tested against a predetermined threshold, $\left(c_{1}+c_{2}\right) / 2>h_{0}$. In case the test is positive a warning is applied. If the flags indicate different under-inflations they are treated separately. Each confidence level is tested against a predetermined threshold $c_{1}>h_{1}$ and $c_{2}>h_{2}$. If any of the tests is positive a warning is applied. If none of the flags indicates underinflation no warning is applied. An important note here is that $h_{1}, h_{2}>h_{0}$, i.e. if both vibration and wheel radius analysis indicate that the same tire (or tires) is underinflated the threshold level is lower. This means that the confidence is higher and smaller pressure changes can be detected faster and more accurate. It also improves the false detection rate. In Figure 9 warning signals for the three different approaches are illustrated for a test run with $30 \%$ under-inflation.

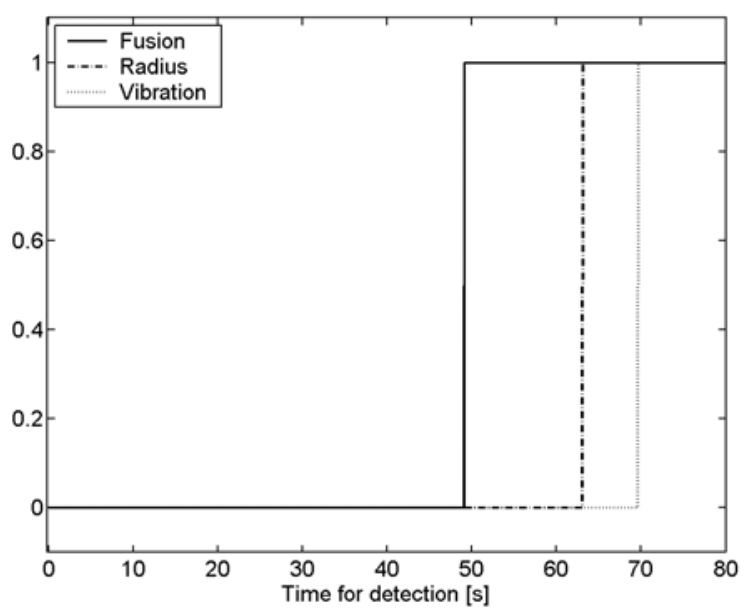

Figure 9: Warning signals for wheel radius analysis, vibration analysis and fusion of both methods. Zero and one means no warning and warning, respectively.

As can be seen in the figure the detection time is shorter when both wheel radius and vibration analysis is used.
The detection time for the fusion algorithm is within 1 minute.

\section{CONCLUSIONS}

A novel approach for detecting low/changed pressure without pressure sensors has been outlined. The approach uses both vibration and wheel radius analysis for optimal performance and only existing sensors in modern vehicles are used. Independently the approaches can detect a pressure loss of $25 \%$ within one minute, but if they are combined a pressure loss of $15 \%$ in one, two, three or four tires (diffusion) can be detected within one minute. To combine the two approaches a voting scheme was proposed.

\section{REFERENCES}

1. F. Gustafsson, M. Drevo, U. Forssell, M. Lofgren, N. Persson, and H. Quicklund, "Virtual sensor of tire pressure and road friction", SAE paper 2001-010796, 2001.

2. F. Gustafsson, S. Ahlqvist, U. Forssell, and N. Persson, "Sensor fusion for accurate computation of yaw rate and absolute velocity", SAE paper 200101-1064, 2001.

3. N. Persson et al., "Tire Pressure Estimation", International patent application WO0187647, 1999.

4. N. Persson and F. Gustafsson, "Event Based Sampling with Application to Vibration Analysis in Pneumatic Tires", in ICASSP 2001.

5. T. Takeyasu et al., "Tire Pneumatic Pressure Detector", EP 700798, 1996.

6. T. Naito et al., "Tyre air pressure estimating apparatus", EP 925960, 1998.

7. F. Braun et al., "Method and apparatus for monitoring the tyre pressure of motor vehicle wheels", EP 938987, 1999.

8. F. Gustafsson, "Slip-based estimation of tire-road friction", Automatica, 33(6), 1087-1099, 1997.

9. J.Y. Wong, "Theory of ground vehicles", $2^{\text {nd }}$ ed. John Wiley and Sons Inc., 1993.

10. T.D. Gillespie, "Fundamentals of Vehicle Dynamics", SAE International, 1992.

11. SchraderBridgeport Standard Thomson. http://www.schrader-bridgeport.net/sld004.html, 2001-11-26.

12. Docket Management System, Docket number NHTSA-2000-8572. http://dms.dot.gov/, 2001-11-26.

\section{CONTACT}

Contact author: Niclas Persson, M.Sc., Linköping University, Dept. of E.E., SE-58183 Linköping, Sweden.

Phone: +46 (0)13 286697. Mobile: +46 (0)733 580103. Fax. +46 (0)13 282622. E-mail: persson@isy.liu.se. URL: $\quad$ www.comsys.isy.liu.se/ persson. 